\title{
ROBUST ESTIMATORS IN FUZZY LOGIC CELLULAR-AUTOMATA SALT AND PEPPER NOISE FILTERING
}

\author{
Nicholas LaVigne ${ }^{(1)}$, Yusuf Bilgic ${ }^{(2)}$ \\ (1) Senior Math Student, Department of Mathematics, SUNY Geneseo, NY, USA \\ ${ }^{(2)}$ Assistant Professor, Department of Mathematics, SUNY Geneseo, NY, USA
}

\begin{abstract}
In order to increase the performance of computational algorithms in terms of efficiency of estimators, we tested new nonparametric estimators in fuzzy and cellular automata models. In particular, image de-noising algorithms exist to restore digital images corrupted by impulse noise. These algorithms may do poorly in many common cases, for example, when high contrast and sharp edges lead to outliers, spikes, or non-symmetric patterns for neighborhing pixels. This would stem from the choices of estimators in the algorithms. We investigated new nonparametric estimators and compared to existing methods in simulation study. We detected better performances of our new methods under various situations.
\end{abstract}

\section{Indexing terms/Keywords}

Nonparametric statistics; Fuzzy Models; Cellular Automata; Image de-noising

\section{TYPE (METHOD/APPROACH)}

Simulation study

\section{Council for Innovative Research}

Peer Review Research Publishing System

Journal: INTERNATIONAL JOURNAL OF COMPUTERS \& TECHNOLOGY

Vol. 15 , No. 1

www.ijctonline.com, editorijctonline@gmail.com 


\section{INTRODUCTION}

Noise filtering is an important aspect of image processing. It has the obvious aesthetic justification of representing clear and precise images but also a practical one in the age of machine learning and computer vision where supervised learning algorithms rely on accurate and uncorrupted sources to train on. Image noise can take on many forms and be caused by errors of transmission, short exposure periods, poor lighting, etc. The particular type considered here is salt \& pepper noise, where pixel values are corrupted randomly to either the maximum or minimum intensity, which, in the black and white case, corresponds to uniformly, distributed black and white dots. For example, as in Figure 1, we see the 256x256 Lena image corrupted with various levels of impulse noise alongside the image our proposed algorithm recovers and the original image itself.
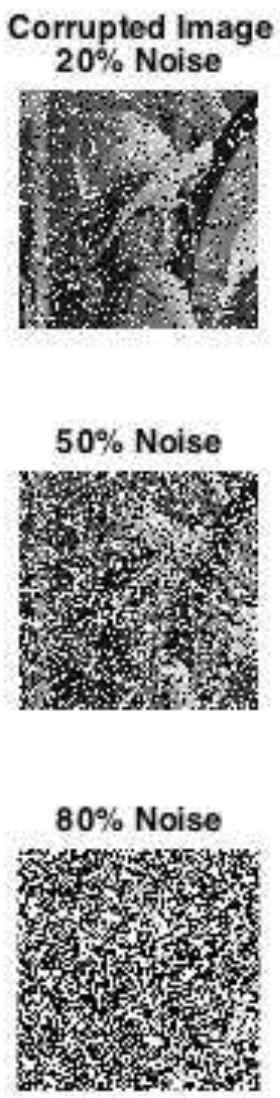

Recovered Image
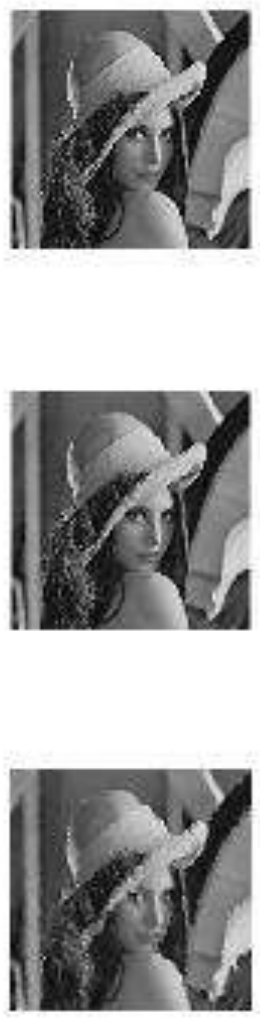

\section{Original image}
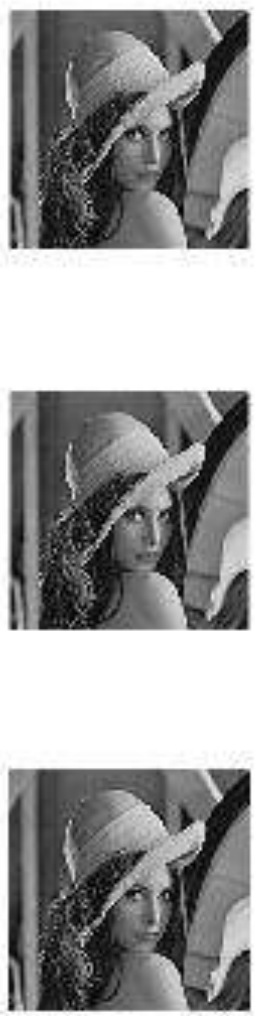

Figure 1. Restored images of Lena with various rate of impulse noise at $\mathbf{2 0} \%, \mathbf{5 0} \%$ and $\mathbf{8 0} \%$

Particularly important to any algorithm proposed to denoise an image is the preservation of edges and clarity of overall features. Edges are important in discerning different objects of an image, such as the difference between handwriting and blank paper or human faces and background. Many algorithms exist to filter salt \& pepper noise, in this paper we consider an existing fuzzy cellular-automata filtering algorithm and test the performance of nonparametric estimators using distributional properties in place of center and spread statistics that rely on more assumptions than might be realistic.

In the next section we provide a brief review of the fuzzy cellular automata filter algorithm for noise elimination. In the methodology section we introduce new nonparametric estimators along with distributional properties to be used in the algorithm. Simulation results are presented in the results section with comparison to the original and other preexisting algorithms.

\section{LITERATURE REVIEW}

Cellular automata (CA) are discrete dynamical systems where behavior is completely determined by local state rules. They were introduced by Von Neumann in 1966 [6] and have since inspired myriads of application across computer science, physics, biology, and many other disciplines. An extensive mathematical review of 2D cellular automata is given in [4] and a study of the use of CA for edge detection in [5]. A 2D CA consists of a uniform grid space where each point is either on or off. After initializing the space the model moves in discrete time steps by updating each point based on a logical computation of the states of its immediate neighbors, known as the local state rule. Different neighborhoods can be 
considered but the most commonly referenced are the Moore neighborhood, consisting of all 8 neighboring points and the Von Neumann neighborhood, consisting of the 4 points directly above below and to the left and right of the index.

A black and white digital image can be represented by a matrix of values between 0 and 255, each representing a single pixel. The eight neighbors of a point $\mathrm{C}_{\mathrm{i}, \mathrm{j}}$, the pixel that represents the central pixel of Moore neighborhood for an image, are shown in a $3 \times 3$ matrix in Table 1 . The values of the neighbors are used to recover the pixel if $C_{i, j}$ is noised. In the $C_{A}$ framework this $3 \times 3$ matrix works like a filter throughout the entire matrix except the edges.

Table 1. A 3x3 filter that includes eight neighbor pixels of a pixel $\mathrm{Cij}$ in an $\mathrm{NxN}$ matrix representation of an image.

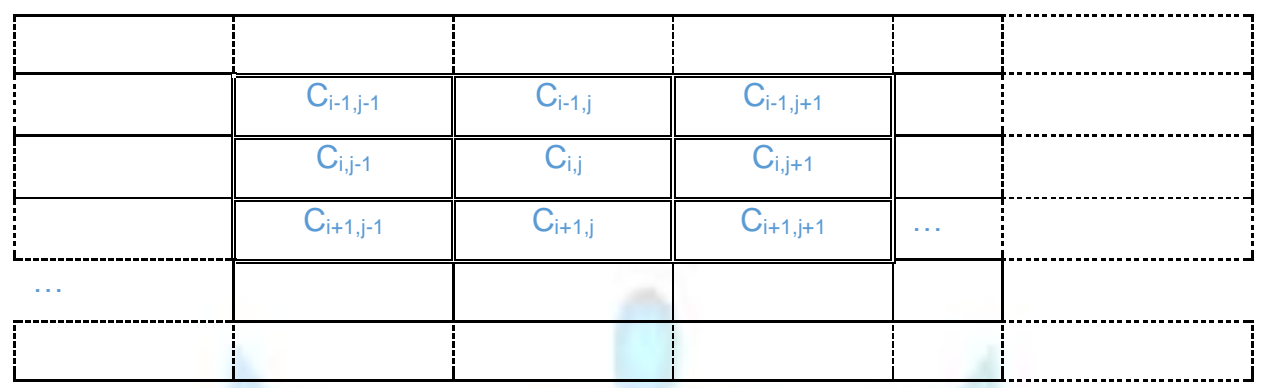

Classical CA are Boolean systems in that pixels can take on only 2 values, on or off, and are updated through logical computations of neighboring values. For the image representations with values between 0 and 255 the logical state rules are replaced by fuzzy logic numerical computations that either emulate or expand on those rules. Fuzzy means here that it is an approach to computing based on many sophisticated options and useful in expert systems such as artificial intelligence applications.

The mathematical basis for a fuzzy cellular automata and a state rule designed for salt \& pepper noise filtering is described in [3]. The particular algorithm is applied to black and white images but could be generalized to multidimensional arrays that represent color images. The algorithm takes an $n \times m$ pixel image corrupted by salt \& pepper noise and proceeds through each pixel, first determining if the pixel is likely to be corrupted and then updating the pixel based a state rule $F$. A pixel $C_{i, j}$ with Moore neighborhood $C_{i \pm 1, j \pm 1}$ is considered corrupted by salt \& pepper noise if it is equal to 0 or 255 and the state rule is given by

$$
F\left(C_{i, j}\right)=\begin{array}{cc}
k_{C_{i, j}} & \text { At least one neighbor } 0 \text { or } 255 \\
127 & \text { All neighbors } 0 \text { or } 255 \\
p_{C_{i, j}} & \text { Otherwise }
\end{array}
$$

Note that if all neighborhood pixels are corrupted then the state rule merely guesses 127 , as it is halfway between 0 and 255. If at least one neighbor is corrupted then $F$ uses an average of the minimum and maximum of the non-corrupted pixels, $k_{C_{i, j}}=\frac{\min \{\text { Neighbors } 0\}+\max \{\text { Neighbors } 255\}}{2}$ (call it $\mathrm{k}$ function). The $\mathrm{k}$ function might be replaced with other options such as the mean and median values of non-corrupted pixels.

If all neighborhood pixels are uncorrupted then the algorithm updates the pixel as a weighted average $p_{C_{i, j}}=\frac{N_{k} \times C_{k}}{N_{k}}$ ( $\mathrm{p}$ function), where the weight of a pixel with value $x$ come from a Gaussian membership function $G(x)=e^{\frac{(x)^{2}}{2^{2}}}$ (G function), where and are the mean and standard deviation of the neighboring pixel values, respectively, which are estimated with user choices.

In the literature, there are several common median filter methods in recovering salt and pepper noise such as the adaptive median filter [11] and the efficient decision-based algorithm of Srinivasan and Ebenezer [10]. Other nonparametric estimates such as percentile and rank-based are missing. Sahin et.al [3] have demonstrated the competitive image recovery of their algorithm as compared with others in the literature based on the Peak Signal to Noise Ratio (PSNR) computed from the Mean Squared Error (MSE) for noise rates as high as $80 \%$. A higher PSNR indicates a higher level of recovery.

In this paper, the distributional properties of the actual pixel neighborhoods as well as new nonparametric estimators are employed in the fuzzy logic CA framework as alternatives to normal distribution assumption and its classical estimators such as mean and standard deviation. Nonparametric estimators have better breakdown values that make the algorithm robust. These are robust to outliers and better performing estimators for skewed or heavy-tailed distributions. 
Consequently, many assumptions can now be relaxed, such as the assumption that neighboring pixels are normally distributed.

\section{METHODOLOGY}

There are several unstated assumptions in the choice of a weighting function which uses the Gaussian distribution that we investigate and propose solutions to here.

\section{Nonparametric Bent Rule for the Shape of a Distribution}

The choice of a Gaussian membership function assumes that neighboring pixel values are symmetrically distributed and have moderate tails. However, it is intuitive that in edge cases the distribution of neighborhood points will likely be skewed and fat tailed. To remedy this we replace the Gaussian function with a skew normal weighting function $S N(x)=\frac{2}{x} \div \quad \frac{x}{-} \div$ (call it SN function), where and are the probability distribution function (pdf) and

cumulative distribution function (cdf) of the normal distribution, respectively. Then, is a location parameter, a scale parameter, and a skew parameter which are to be estimated by user choices. To estimate we utilize a nonparametric scheme from McKean \& Kloke [1] to classify these distributions in terms of skewness and kurtosis based on the bent scores $Q_{1}$ and $Q_{2}$ respectively,

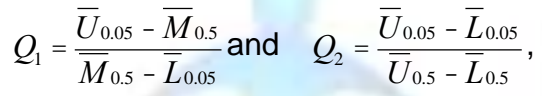

where $\bar{U}_{x}, \bar{M}_{x}$, and $\bar{L}_{x}$ denote the means of the upper, middle, and lower $x \rtimes 00$ th percentile. Then is interpolated from a table of recommended values for different values of $Q_{1}$ and, $Q_{2}$ from a simulation study in [1].

\section{New Nonparametric Estimators}

The use of mean and standard deviation for estimating location and spread parameters of the neighborhoods for use in the state rule might not be robust in the face of an outlier in a small sample (8 pixel values in the Moore neighborhood) and skewed distributions. Therefore, we are motivated to introduce robust estimators of both location (center) and spread (scale) parameters as user choices. In particular we considered the median and Hodges-Lehman estimator (HL) for measures of centrality, and the interquartile range (IQR), median absolute deviation (MAD), and dispersion type (Disp) for measures of spread.

For an arbitrary sample vector $V=\left\{V_{1}, V_{2}, \square, V_{m}\right\}$, the location estimate associated with the linear scores is the well-known Hodges-Lehmann estimator, $H L(V)=$ med $_{j k} \frac{V_{j}+V_{k}}{2}$ This is the estimator associated with the signed-rank Wilcoxon scores so it has a high breakdown point, i.e., the presence of corrupted/outliers in up to $30 \%$ of the data points in a sample don't affect the location estimate and this makes the estimator robust [1,9]. The median absolute deviation (MAD) is defined as $M A D(V)=1.483 \times \operatorname{med}_{i}\left|V_{i} \operatorname{med}_{j}\left\{V_{j}\right\}\right|$ where med is the median. Hettmansperger \& McKean [9] defines the functional corresponding to the dispersion type of estimate for linear scores for a vector $V$ as $\operatorname{Disp}(V)=\frac{2 \sqrt{ }}{m}{ }_{i=1}^{m} \frac{\operatorname{Rank}\left(V_{1}\right)}{m+1} \frac{1}{2} \div \bigvee_{i}$. These estimators are consistent location and spread functions under a Gaussian distribution.

We pair location and spread estimators in the algorithm as follows: median with IQR, median with MAD, and HL with Disp.

\section{Simulation Setup}

In our simulation study for the fuzzy CA algorithm, we used various types of estimators for k, $\mathrm{G}$ and $\mathrm{SN}$ functions. The $\mathrm{k}$ function has four options: original (1), mean (2), median (3), and HL (4). The weighting functions (G and SN) also have four options for parameter estimation: mean-sd (1), median-IQR (2), median-MAD (3), and HL-Disp (4). In our outputs, we use these labels for changes to different parts of the algorithm, G represents the Gaussian weighting function and SN the skew normal, followed by the estimator used in the $k$ function and then the pair of estimators used in the weighting function. For example, SN 2-3 is the state rule that uses the mean of non-corrupted pixels in $\mathrm{k}$ function and then the pair of median and MAD as location and spread estimates for the skew normal weighting function in the SN function. In total, between modifying the $\mathrm{k}$ function, the $\mathrm{p}$ function via the weighting function used, and the $\mathrm{p}$ function via the estimator pairs, we have a total of 32 different combinations to test including the original formulation.

We selected several combinations of our proposed estimators and ran simulations on the 256x256 black and white Lena image. We make comparison of our method with the method in Sahin et al. [3] since their method overall outperforms many other existing algorithms including Chan et.al [7] and Wang [8]. 


\section{RESULTS}

The computed PSNR for several cases of the modified algorithm on the Lena image for simulated noise ratios from 20 $80 \%$ are shown in Figure 2. We can see that for ratios less than 50\% the modifications (G 3-2), (G 4-2), and (G 4-4) all outperform the original by $0.5-1$ which increased the efficiency of the estimators from $12 \%$ to $26 \%$ (relative efficieny of their MSE`s). After $50-60 \%$ noise this improvement is lost. The modifications shown that utilize the skew-normal weighting function are largely on par with the original, as is the case with (SN 2-3). The modifications (SN 2-3), (G 4-2) and (G 4-4) are promising overall.

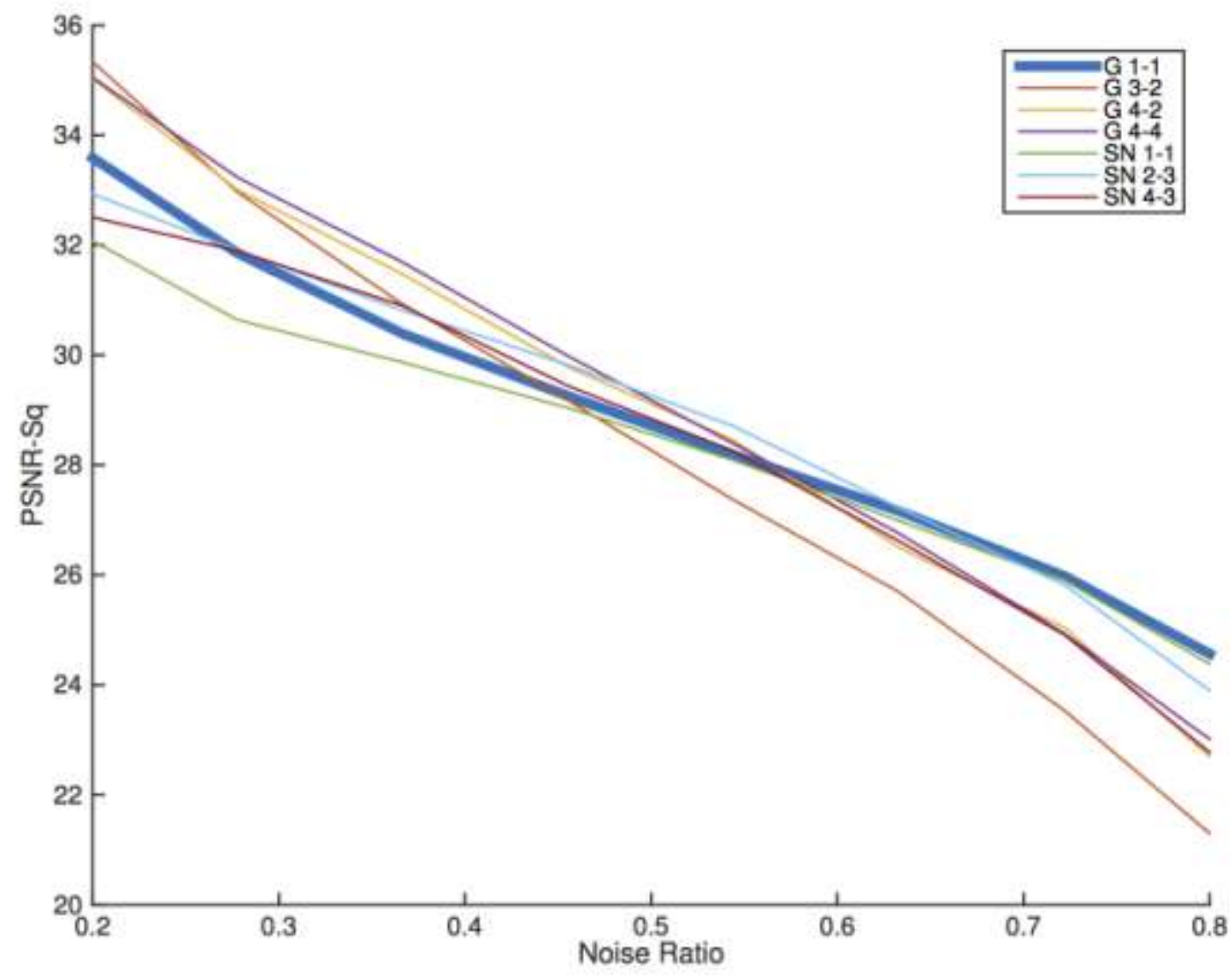

\section{Figure 2. PSNR graphs for the $256 \times 256$ Lena image with $3 \times 3$ filter against noise ratio for our proposed methods tests along with the original algorithm (G 1-1) in Sahin et al.}

The notable modifications we introduce also outperform the other common salt \& pepper noise filtering algorithms from [7] and [8] on the Lena image. Again, for low noise ratios we observed that the modification (G 4-2) has a higher PSNR than the original and the other algorithms.

In attempts to increase the neighborhood sample size and, therefore, the performance of the robust estimators, a $5 \times 5$ filter was tested as well. While it did increase the margin of improvement between the original algorithm and our modifications the increase was only relative and decreased the overall performance of the algorithm Differences in computation time between the original algorithm and our modifications were neglible for 256x256 and other larger images.

\section{DISCUSSION}

Our preliminary results suggest the better performance of some combinations of nonparametric estimators in place of $k$ function and location-scale estimator pairs in the weighting function for $p$ function in the fuzzy cellular-automata state rule $F$. The drop in performance after $60 \%$ is likely due to the very few non-corrupted pixels remaining. In the $60 \%$ case the expected number of uncorrupted pixels in an 8 element Moore neighborhood is only 3.2. Thus the algorithm is mainly relying on the $\mathrm{k}$ function and an average is more accurate just in terms of smoothing on such small sample sizes. Related to this there is the potential to use neighbor information, in the form of spatial estimators, which we left for future study.

The skew-normal weighting function did not turn out to increase performance in most cases. We hypothesize that this is due to the choice of scale multipliers, which came from recommendations based on a normal distribution. The increased sample size from the $5 \times 5$ filter did not improve overall results likely because the distributions now encompassed an area 
with potentially more features that were combined together as opposed to the highly local Moore neighborhood. However, it was interesting to see increased relative performance due to the increased sample size.

To better understand the behavior of these modifications future directions include a simulation study across all 32 possible combinations and with multiple images. Other nonparametric estimator alternatives to the fuzzy CA algorithm that uses mean and standard deviation can be applied to improve in the next studies.

\section{REFERENCES}

[1] Kloke, John, and Joseph W. McKean. Nonparametric statistical methods using R. CRC Press, 2014.

[2] McKean, Joseph W., and John D. Kloke. "Efficient and adaptive rank-based fits for linear models with skewnormal errors." Journal of Statistical Distributions and Applications 1.1 (2014): 1-18.

[3] Sahin, Ugur, Selman Uguz, and Ferat Sahin. "Salt and pepper noise filtering with fuzzy-cellular automata." Computers \& Electrical Engineering 40.1 (2014): 59-69.

[4] Uguz, Selman, et al. "Self-replicating patterns in 2D linear cellular automata." International Journal of Bifurcation and Chaos 24.01 (2014): 1430002.

[5] Uguz, Selman, Ugur Sahin, and Ferat Sahin. "Uniform cellular automata linear rules for edge detection." Systems, Man, and Cybernetics (SMC), 2013 IEEE International Conference on. IEEE, 2013.

[6] Von Neumann, J. and A. W. Burks. Theory of self-reproducing automata. Urbana, University of Illinois Press. 1966.

[7] Chan, Raymond H., Chung-Wa Ho, and Mila Nikolova. "Salt-and-pepper noise removal by median-type noise detectors and detail-preserving regularization". Image Processing, IEEE Transactions on 14.10 (2005): 14791485.

[8] Wang, Shuenn-Shyang, and Cheng-Hao Wu. "A new impulse detection and filtering method for removal of wide range impulse noises." Pattern Recognition42.9 (2009): 2194-2202.

[9] Hettmansperger, T. P. and McKean, J. W. Robust nonparametric statistical methods. 2nd Ed., New York:Chapman-Hall. 2011.

[10] Srinivasan KS, Ebenezer D. A new fast and efficient decision-based algorithm for removal of high-density impulse noises. IEEE Signal Process Letter, 2007;14:189-92.

[11] Hwang H, Haddad RA. Adaptive median filters: new algorithms and results. IEEE Trans Image Process 1995; 4(4):499-502.

\section{Author's biography with Photo}

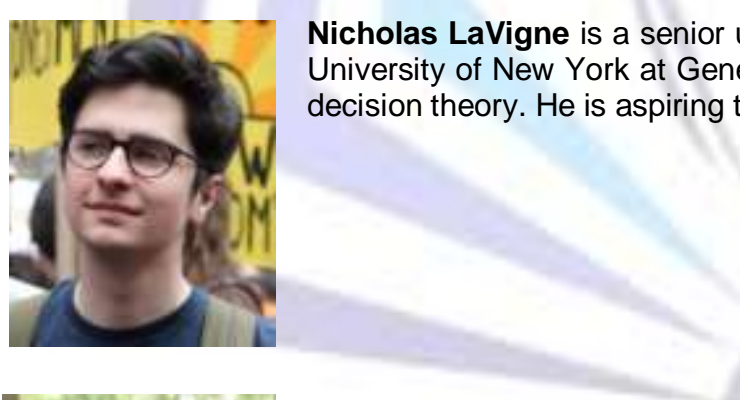

Nicholas LaVigne is a senior undergraduate student in the department of mathematics at the State University of New York at Geneseo. His interests include applied probability, complex systems, and decision theory. He is aspiring to enroll in a PhD program in applied mathematics.

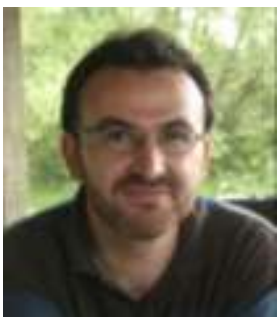

Yusuf Bilgic received his Ph.D. at Western Michigan University (WMU), USA in 2012. Since then, he has been working at State University of New York (SUNY Geneseo) in USA, Department of Mathematics. His current research interests includes nonparametric methods in statistical analysis. $\mathrm{He}$ is the author of an $\mathrm{R}$ package, rlme, on rank-based analysis of random effects models for hierarchical designs.

Notes:

We classified the distributions of neighborhoods (for $n=8$ ) as either symmetric, right or left skewed, with either light, medium, or heavy tails using the nonparametric methods. In our experiment, we observed a large proportion of skewed and fat tailed neighborhoods in images based on this classification. 